https://helda.helsinki.fi

\title{
Transuranium compounds probed by nonresonant inelastic x-ray scattering
}

Sundermann, M.

2020-02-03

Sundermann , M , Simonelli , L , Huotari , S , Eloirdi , R , Lander , G H , Caciuffo , R \& van der Laan , G 2020 , ' Transuranium compounds probed by nonresonant inelastic x-ray scattering ' , Physical Review B , vol. 101 , no. 7 , 075103 . https://doi.org/10.1103/PhysRevB.101.075103

http://hdl.handle.net/10138/312382

https://doi.org/10.1103/PhysRevB.101.075103

unspecified

publishedVersion

Downloaded from Helda, University of Helsinki institutional repository.

This is an electronic reprint of the original article.

This reprint may differ from the original in pagination and typographic detail.

Please cite the original version. 


\title{
Transuranium compounds probed by nonresonant inelastic x-ray scattering
}

\author{
M. Sundermann, ${ }^{1,2}$ L. Simonelli, ${ }^{3,4}$ S. Huotari, ${ }^{5}$ R. Eloirdi, ${ }^{6}$ G. H. Lander, ${ }^{6}$ R. Caciuffo $\odot,{ }^{6}$ and G. van der Laan $\odot^{7, *}$ \\ ${ }^{1}$ Institute of Physics II, University of Cologne, Zülpicher Straße 77, D-50937 Cologne, Germany \\ ${ }^{2}$ Max Planck Institute for Chemical Physics of Solids, Nöthnizer Straße 40, 01187 Dresden, Germany \\ ${ }^{3}$ European Synchrotron Radiation Facility (ESRF), B.P. 220, 38043 Grenoble Cédex, France \\ ${ }^{4}$ ALBA Synchrotron Light Source, E-08290 Cerdanyola del Vallès, Barcelona, Spain \\ ${ }^{5}$ Department of Physics, P.O. Box 64, FI-00014 University of Helsinki, Finland \\ ${ }^{6}$ European Commission, Joint Research Centre (JRC), Directorate for Nuclear Safety and Security, \\ Postfach 2340, D-76125 Karlsruhe, Germany \\ ${ }^{7}$ Magnetic Spectroscopy Group, Diamond Light Source, Didcot OX11 ODE, United Kingdom
}

(Received 20 December 2019; revised manuscript received 17 January 2020; accepted 21 January 2020; published 3 February 2020)

\begin{abstract}
While x-ray absorption spectroscopy is mainly governed by electric-dipole transitions, the technique of nonresonant inelastic x-ray scattering (NIXS) offers the possibility to explore higher-order multipole transitions. These transitions obey different selection rules that can reach final states of higher angular momenta, opening complementary spectroscopic perspectives. Here, we investigate the suitability of NIXS to study transuranium compounds. We show that the $K$ edge of the Be encapsulation can be practically fully excluded by using the imaging capabilities of the technique arising from the position of the signals on the multidetector. Experimental results for the multipole transitions at the actinide $O_{4,5}$ edges (90-120 eV) in $\mathrm{UO}_{2}, \mathrm{NpO}_{2}, \mathrm{PuO}_{2}$, and $\mathrm{Pu}_{2} \mathrm{O}_{3}$ are compared with multielectronic calculations. The spectral features are shown to be very sensitive to the ratio of the triakontadipole and octupole transitions, which could potentially be used to assess the radial expansion of the $5 f$ wave function, which is expected to occur in covalent mixing with the $\mathrm{O} 2 p$ states.
\end{abstract}

DOI: 10.1103/PhysRevB.101.075103

\section{INTRODUCTION}

X-ray absorption spectroscopy (XAS) has been established as a powerful method to obtain detailed information about the local electronic ground state of materials by direct excitation of a core electron into an unoccupied valence state [1]. In particular, rich multiplet structure is present at the $L_{2,3}$ edges ( $2 p \rightarrow 3 d$ excitation) of $3 d$-transition metals [2], the $M_{4,5}(3 d \rightarrow 4 f)$ of lanthanides [3], and the $M_{4,5}$ $(3 d \rightarrow 5 f)$ and $N_{4,5}(4 d \rightarrow 5 f)$ of actinides [4], providing a direct fingerprint for the electronic ground state. More intricate to analyze are those XAS spectra where core and valence states share the same principal quantum number, such as in the $M_{2,3}$ edge ( $3 p \rightarrow 3 d$ excitation) of $3 d$-transition metals [5], the $N_{4,5}(4 d \rightarrow 4 f)$ of lanthanides [6], and the $O_{4,5}(5 d \rightarrow 5 f)$ of actinides $[7,8]$. These shallow core levels give rise to very intense but broad peaks, known as giant or Fano resonances [9]. In the case of the electric-dipole transition $5 d \rightarrow 5 f$ in actinides, the asymmetric Fano profile originates from the excitation-decay process $5 f^{n}+\hbar \omega \rightarrow$ $5 d^{9} 5 f^{n+1} \leftrightarrow 5 d^{10} 5 f^{n-1} \varepsilon \ell$ in resonance with the direct photoemission $5 f^{n}+\hbar \omega \rightarrow 5 d^{10} 5 f^{n-1} \varepsilon \ell$, where $\epsilon \ell$ denotes a continuum state. The super-Coster-Kronig decay of the excited state $5 d^{9} 5 f^{n+1}$ is due to the large radial matrix element $R(5 d, \varepsilon \ell ; 5 f, 5 f)$. Because of the large $5 d-5 f$ Coulomb and exchange interactions, the multiplet structure in the final state stretches out over an energy of $\sim 20 \mathrm{eV}$ [8]. Dipole transitions

${ }^{*}$ Corresponding author: gerrit.vanderlaan@diamond.ac.uk are only allowed to final states with relatively low angular momentum, which are primarily located at the high-energy side of the manifold $[10,11]$. For Th, $\mathrm{U}$, and $\mathrm{Np}$ the dipole transitions in XAS and electron-energy-loss spectroscopy (EELS) result in a broad peak accompanied by small prepeaks at $\sim 15 \mathrm{eV}$ lower energy [8]. The prepeaks are allowed due to first-order perturbation by the $5 d$ spin-orbit interaction on the $5 d-5 f$ exchange interaction, where the latter interaction mainly determines the energy splitting [12]. Generic prepeaks also occur at the lanthanide $N_{4,5}$ edges [6] and $3 d$-transition metal $M_{2,3}$ edges [5].

For higher-order multipole transitions the selection rules allow reaching higher orbital momenta, which are located at lower energies, below the onset of the continuum states. These transitions will become observable in nonresonant inelastic $\mathrm{X}$ ray scattering (NIXS), also known as x-ray Raman scattering spectroscopy, using high photon energies $(\sim 10 \mathrm{keV})$ with a large momentum transfer $\left(\sim 10 \AA^{-1}\right)$. While the cross section of NIXS is rather small, it is substantially larger for transitions between shells of the same principal quantum number, like the $5 d \rightarrow 5 f$, due to the large radial overlap. The high momentum transfer also moves the Compton peak to higher energies so that nondipole signals of these transitions are more easily observed. However, most importantly a high momentum transfer increases the nondipolar excitation signal strongly owing to the large increase of its transition matrix element with increasing $q$. Currently, there are only a small number of synchrotron radiation facilities around the world with the combination of high incident flux and a highly multiplexed spectrometer where these measurements are feasible. 
The penetration length $\left(e^{-1}\right)$ for $\mathrm{x}$ rays of $10 \mathrm{keV}$ is $\sim 5 \mu \mathrm{m}$, which makes NIXS sensitive to the top $\sim 2.5 \mu \mathrm{m}$ of the sample. This is much larger than the extent of any surface effects, so this probe may truly be considered as a bulk probe. In contrast, in soft $\mathrm{x}$-ray absorption the sampling depth is $\sim 3 \mathrm{~nm}$ (total-electron yield) or $\sim 5 \mathrm{~nm}$ (fluorescence yield) at the $O_{4,5}$ edge of $\mathrm{UO}_{2}$. This high absorbance also leads to strong saturation and self-absorption effects [1]. The bulk sensitivity of hard $\mathrm{x}$ rays offers a considerable advantage over soft $\mathrm{x}$ rays, and permits spectroscopy under extreme conditions, e.g., in high-pressure cells, as well as the study of samples, such as actinides, that must be encapsulated for safety reasons. Moreover, we will demonstrate here that the contribution of any encapsulation can be mostly removed from the signal.

NIXS yields new spectroscopic features, which have been previously described theoretically and successfully compared with experiments $[11,13-26]$. Strong NIXS signals have been observed at the $O_{4,5}$ of $\mathrm{UO}_{2}$, USe, and US [20], and similarly at the $N_{4,5}$ edges of rare-earth materials [21]. Several U compounds have subsequently been investigated [19,25,27]. Recently, directional dichroism of the NIXS spectra was used to probe the crystal-field interaction in a $\mathrm{UO}_{2}$ single crystal [28]. While so far only Th and U compounds have been measured, calculations of the $O_{4,5}$ NIXS spectra for all actinides from Th $5 f^{0}$ to Cf $5 f^{9}$ have already been published [11].

Actinide-based oxides constitute the main part of longlived nuclear waste. A comprehensive knowledge of their physical and chemical properties therefore remains a key topic of condensed matter science. The nature and extent of the highest oxidation state in solid-state actinide compounds are still unexplored compared with those of small molecules [29]. NIXS measurements would be of particular interest here.

In this paper we report experimental results of spectra beyond $\mathrm{U}$, namely for $\mathrm{Np}$ and $\mathrm{Pu}$ oxides. Comparison to the calculational results shows a good agreement for the $5 d \rightarrow 5 f$ spectra and allows us to distinguish between various valence states.

The dioxides, $A n \mathrm{O}_{2}(A n=\mathrm{U}, \mathrm{Np}, \mathrm{Pu})$, crystallize in the well-known $\mathrm{CaF}_{2}$ fluorite structure, with eight-coordinated $A n$ and four-coordinated $\mathrm{O}$. For divalent oxygen, the stoichiometry implies a formally $5 f^{2}, 5 f^{3}$, and $5 f^{4}$ configuration and a $\Gamma_{5}$ triplet, a $\Gamma_{8}$ quartet, and a $\Gamma_{1}$ singlet crystal-field ground state for $\mathrm{U}^{4+}, \mathrm{Np}^{4+}$, and $\mathrm{Pu}^{4+}$, respectively [30-33].

Unquenched orbital degrees of freedom combined with strong spin-orbit coupling and higher-order electromagnetic multipole interactions are a source of complexity in actinide oxides [34]. These interactions may drive exotic phase transitions with nondipolar (hidden) order parameters. A prominent example is $\mathrm{NpO}_{2}$, where a phase transition at $25 \mathrm{~K}$ stabilizes a noncollinear 3-k order of rank-5 magnetic multipoles (triakontadipole), with electric quadrupoles as a secondary order parameter $[35,36] . \mathrm{UO}_{2}$, on the other hand, displays a first-order phase transition at $30.8 \mathrm{~K}$ to a noncollinear dipolar antiferromagnetic (AF) 3-k structure with a simultaneous electric-quadrupole ordering of the charge density [37]. The collective dynamics of the system is also strongly influenced by multipolar interactions and can include fluctuations of quadrupoles and higher-rank multipoles, alone or in combination with dipoles $[38,39]$.
$\mathrm{PuO}_{2}$ is an insulator with a temperature-independent magnetic susceptibility [40] and a vanishing local magnetic moment [41]. This nonmagnetic behavior is reproduced by firstprinciples calculations yielding a nonmagnetic singlet ground state characterized by a noninteger filling of the plutonium $5 f$ shell $\left(n_{f} \simeq 4.5\right)$ [42].

The hexagonal form of plutonium sesquioxide $\beta-\mathrm{Pu}_{2} \mathrm{O}_{3}$ crystallizes in the $\mathrm{La}_{2} \mathrm{O}_{3}$ structure [43]. This compound orders $\mathrm{AF}$ at $19 \mathrm{~K}$, with magnetic and chemical unit cells becoming identical below $4 \mathrm{~K}$. The magnetic moment of $0.60(2) \mu_{\mathrm{B}}$ per $\mathrm{Pu}$ ion is oriented along the $c$ axis and is consistent with a Kramers doublet $| \pm 3 / 2\rangle$ ground state of the $\mathrm{Pu}^{3+}$ ion [43]. A few attempts to calculate the electronic structure of $\mathrm{Pu}_{2} \mathrm{O}_{3}$ have been performed, suggesting a localized nature of the $5 f$ electrons and a semiconducting electronic structure [44-47].

\section{THEORETICAL}

\section{A. General theory}

NIXS is a photon-in $\left(\hbar \omega_{i}\right)$, photon-out $\left(\hbar \omega_{f}\right)$ process, e.g., in the case of the $O_{4,5}$ excitation given by the scattering process $5 f^{n}+\hbar \omega_{i} \rightarrow 5 d^{9} 5 f^{n+1}+\hbar \omega_{f}$. The double differential cross section for NIXS is

$$
\frac{d^{2} \sigma}{d \Omega d \omega}=\left(\frac{d \sigma}{d \Omega}\right) S(\mathbf{q}, \omega),
$$

where $\hbar \omega=\hbar \omega_{i}-\hbar \omega_{f}$ is the energy loss and $\mathbf{q}=\mathbf{k}_{i}-\mathbf{k}_{f}$ is the scattering vector with wave vectors $\mathbf{k}_{i}$ and $\mathbf{k}_{f}$ of the incident and scattered radiation, respectively, and $(d \sigma / d \Omega)$ is the Thomson scattering cross section [11]. The dynamic structure factor from initial state $\psi_{i}$ to final state $\psi_{f}$ with energies $E_{i}$ and $E_{f}$ can be written as

$$
S(\mathbf{q}, \omega)=\sum_{f}\left|\left\langle\psi_{f}\left|e^{i \mathbf{q} \cdot \mathbf{r}}\right| \psi_{i}\right\rangle\right|^{2} \delta\left(E_{i}-E_{f}+\hbar \omega\right) .
$$

The transition operator can be expanded as

$$
e^{i \mathbf{q} \cdot \mathbf{r}}=\sum_{k=0}^{\infty} \sum_{m=-k}^{k} i^{k}(2 k+1) j_{k}(q r) C_{m}^{(k) *}(\hat{\mathbf{q}}) C_{m}^{(k)}(\hat{\mathbf{r}}),
$$

where $C_{m}^{(k)}(\hat{\mathbf{q}})=\sqrt{4 \pi /(2 k+1)} Y_{m}^{(k)}\left(\theta_{q}, \phi_{q}\right)$ are the renormalized spherical harmonics of rank $k$ with components $m$ and $\theta_{q}, \phi_{q}$ are the polar and azimuthal angles of $\hat{\mathbf{q}}$. The $j_{k}(q r)$ are spherical Bessel functions of rank $k$. Substitution gives

$$
S(\mathbf{q}, \omega)=\sum_{f} \sum_{k=0}^{\infty} I^{k}\left(\omega, \theta_{q}, \phi_{q}\right)\left|\left\langle\psi_{f}(\mathbf{r})\left|j_{k}(q r)\right| \psi_{i}(\mathbf{r})\right\rangle\right|^{2} .
$$

Using that the radial expansion is linearly independent from the direction, i.e., $\psi(\mathbf{r})=R(r) \psi(\theta, \phi)$, and assuming that the cross section does not vary much for changes of $\Delta q / q \approx$ $\frac{1}{2} \Delta E_{\text {edge }} / \hbar \omega_{i} \approx 0.0005$ (see Fig. 1 ), $S(\mathbf{q}, \omega)$ can be separated into an angular and radial part. Then the angular part of the $2^{k}$-pole spectrum is

$$
\begin{aligned}
I^{k}\left(\omega, \theta_{q}, \phi_{q}\right)= & (2 k+1)^{2} \sum_{f} \sum_{m=-k}^{k} \mid\left\langle\psi_{f}(\hat{\mathbf{r}})\right| C_{m}^{(k) *}(\hat{\mathbf{q}}) \\
& \times\left. C_{m}^{(k)}(\hat{\mathbf{r}})\left|\psi_{i}(\hat{\mathbf{r}})\right\rangle\right|^{2} \delta\left(E_{i}-E_{f}+\hbar \omega\right) .
\end{aligned}
$$




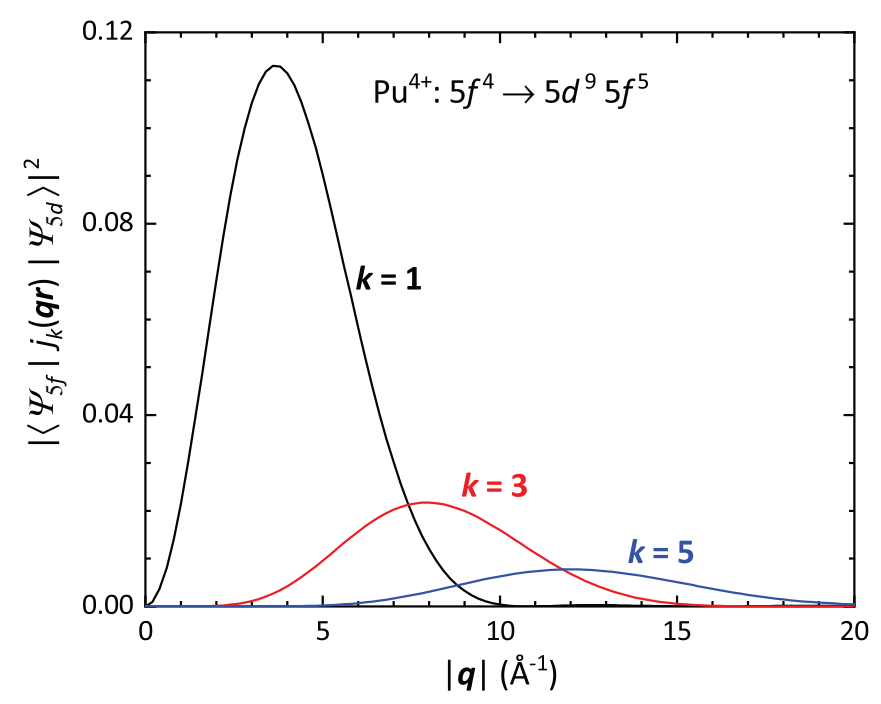

FIG. 1. The $q$ dependence of the radial cross section $\left|\left\langle\psi_{5 f}(r)\left|j_{k}(q r)\right| \psi_{5 d}(r)\right\rangle\right|^{2}$ for the multipole channels $k=1,3$, 5 in the $\mathrm{Pu}^{4+} 5 d \rightarrow 5 f$ NIXS process. The curves for $\mathrm{U}$ and $\mathrm{Np}$ are very similar.

Of course, there are also interference terms between the different channels $\left(k \neq k^{\prime}\right)$, which the calculation takes into account, but these disappear in the isotropic spectrum.

The matrix element in Eq. (5) restricts the multipole moments $k$ in the transition between the orbital angular moments $\ell_{i}$ and $\ell_{f}$ by the triangle condition, $\left|\ell_{i}-\ell_{f}\right| \leqslant k \leqslant \ell_{i}+\ell_{f}$, and the parity rule, $\ell_{i}+\ell_{f}+k=$ even. Thus for the $d \rightarrow$ $f$ transitions, $k=1$ (dipole), $k=3$ (octupole), and $k=5$ (triakontadipole) transitions are allowed. The weight of each channel is determined by the square of the radial matrix element $\left\langle\psi_{5 f}(r)\left|j_{k}(q r)\right| \psi_{5 d}(r)\right\rangle$, cf. Eq. (4). For increasing multipole order $k$, the first largest peak of the spherical Bessel function $j_{k}(q r)$ occurs at successively higher values of $q r$, and decreases in absolute intensity. The range of $q r$ that contributes most is determined by the extent of the atomic radial wave functions $\psi_{5 f}(r)$ and $\psi_{5 d}(r)$. Figure 1 shows the radial cross sections calculated for the $\mathrm{Pu}^{4+} O_{4,5}$ NIXS, using Cowan's code [48], which confirms that the maxima for the three channels are at very different values of $q$. The cross section for the dipole transition $(k=1)$ is suppressed with increasing $q$, while those of the $k=3$ and $k=5$ become enhanced at higher $q$ values, respectively. As seen in Fig. 1 the intensities of the $k=3$ and 5 transitions become larger compared to $k=1$ for $q>8 \AA^{-1}$, which requires hard x rays with large scattering angles.

\section{B. Calculational method}

Spectral simulations were performed in intermediate coupling for the actinide compounds listed in Table I using the full-multiplet code QUANTY [49]. The methodology for calculating the actinide NIXS spectra, with electrostatic and spin-orbit interactions treated on an equal footing, is discussed in Ref. [11].

For the ground state $[\mathrm{Rn}] 5 f^{n}$ in spherical symmetry without crystal field the total momentum $J$ is a good quantum number due to the large $5 f$ spin-orbit interaction, while the $L S$ terms are strongly mixed. The atomic parameters, calculated using Cowan's atomic multiplet code [48], are given in Table II as their unscaled Hartree-Fock values. In the solid state the Slater integrals for the electrostatic interactions require a reduction factor $g$ to account for intra-atomic relaxation effects $[3,8]$. For small spin-orbit interaction, $L S$ coupling gives the Hund's rule ground state ${ }^{3} H_{4},{ }^{4} H_{9 / 2},{ }^{5} I_{4}$, and ${ }^{6} H_{5 / 2}$ for $n=2,3,4,5$, respectively. Other $L S$ states with the same $J$ value are mixed into the ground state by the finite spin-orbit interaction [8,11]. Table I gives the $L S$ composition of the actinide ground states, which not only shows that the mixing increases for reduced values of $g$, but also that the mixing increases for higher $f^{n}$ counts. For instance, taking $g=0.65$, the $\mathrm{U}^{4+} 5 f^{2}$ ground state contains $86.7 \%{ }^{3} \mathrm{H}_{4}$, which is diminished to $54.4 \%{ }^{6} H_{5 / 2}$ for the $\mathrm{Pu}^{3+} 5 f^{5}$ ground state.

Spectra were fitted in two distinct ways: In the first case $g$ was kept constant for all compounds, and in the second case $g$ was varied independently for each compound. The calculated line spectra were convoluted by a Lorentzian line shape of $0.6 \mathrm{eV}$ FWHM and a Gaussian line shape of $1.3 \mathrm{eV}$ FWHM to account for lifetime and instrumental broadening, respectively.

Given the moderate energy resolution of the presented measurement it is unrealistic to extract information about the crystal-field ground state or to vary each Slater integral independently. However, higher energy resolution is already available, paving the way for this $[28,50]$.

\section{EXPERIMENTAL}

Pellets were prepared of $\mathrm{NpO}_{2}(23.8 \mathrm{mg}),{ }^{242} \mathrm{PuO}_{2}$ $(2.3 \mathrm{mg})$, and $\beta{ }^{242} \mathrm{PuO}_{2}(2.2 \mathrm{mg})$ at the Joint Research

TABLE I. Actinide oxides and their electronic configuration. $J$ value and $L S$ term composition of the ground state for the indicated reduction factors $g$ of the $5 f-5 f$ Slater integrals.

\begin{tabular}{|c|c|c|c|c|}
\hline Oxide & Configuration & $g$ & $J$ & $L S$ term composition \\
\hline \multirow[t]{2}{*}{$\mathrm{UO}_{2}$} & \multirow[t]{2}{*}{$\mathrm{U}^{4+} 5 f^{2}$} & 0.65 & 4 & $0.867{ }^{3} H+0.123{ }^{1} G+0.010{ }^{3} F$ \\
\hline & & 0.60 & 4 & $0.860{ }^{3} H+0.129{ }^{1} G+0.011^{3} F$ \\
\hline \multirow[t]{2}{*}{$\mathrm{NpO}_{2}$} & \multirow[t]{2}{*}{$\mathrm{Np}^{4+} 5 f^{3}$} & 0.65 & $\frac{9}{2}$ & $0.786^{4} H+0.190^{2} H+0.022^{2} G+0.0012^{4} F$ \\
\hline & & 0.70 & $\frac{9}{2}$ & $0.801{ }^{4} H+0.179^{2} H+0.019^{2} G+0.0010^{4} F$ \\
\hline \multirow[t]{2}{*}{$\mathrm{PuO}_{2}$} & \multirow[t]{2}{*}{$\mathrm{Pu}^{4+} 5 f^{4}$} & 0.65 & 4 & $0.725^{5} I+0.226{ }^{3} H+0.045^{1} G+0.005^{3} F+0.0001{ }^{5} D$ \\
\hline & & 0.70 & 4 & $0.750{ }^{5} I+0.209^{3} H+0.038{ }^{1} G+0.004{ }^{3} F+0.0001{ }^{5} D$ \\
\hline $\mathrm{Pu}_{2} \mathrm{O}_{3}$ & $\mathrm{Pu}^{3+} 5 f^{5}$ & 0.65 & $\frac{5}{2}$ & $0.544{ }^{6} H+0.307{ }^{4} G+0.123{ }^{2} F+0.024{ }^{2} D+0.0025{ }^{4} P+0.0000{ }^{4} S$ \\
\hline
\end{tabular}


TABLE II. Average energy $\left(E_{\text {av }}\right)$ and calculated unscaled (i.e., $100 \%$ Hartree-Fock) values of the Coulomb $\left(F^{k}\right)$ and exchange $\left(G^{k}\right)$ integrals and the spin-orbit parameters $(\zeta)$ for the $\psi_{i}\left(5 f^{n}\right) \rightarrow$ $\psi_{f}\left(5 d^{9} 5 f^{n+1}\right)$ transitions for the various ionic configurations. All energies in $\mathrm{eV}$.

\begin{tabular}{lrrrr}
\hline \hline & ${ }^{92} \mathrm{U}^{4+} f^{2}$ & ${ }^{93} \mathrm{~Np}^{4+} f^{3}$ & ${ }^{94} \mathrm{Pu}^{4+} f^{4}$ & ${ }^{94} \mathrm{Pu}^{3+} f^{5}$ \\
\hline$E_{\text {av }}$ & 99.5 & 104.4 & 109.3 & 109.3 \\
$\psi_{i}:$ & & & & \\
$\zeta(f)$ & 0.262 & 0.298 & 0.335 & 0.308 \\
$F^{2}(f, f)$ & 9.510 & 9.903 & 10.278 & 9.696 \\
$F^{4}(f, f)$ & 6.222 & 6.486 & 6.738 & 6.314 \\
$F^{6}(f, f)$ & 4.567 & 4.765 & 4.953 & 4.627 \\
$\psi_{f}:$ & & & & \\
$\zeta(f)$ & 0.275 & 0.310 & 0.347 & 0.321 \\
$\zeta(d)$ & 3.248 & 3.477 & 3.756 & 3.724 \\
$F^{2}(f, f)$ & 9.707 & 10.082 & 10.442 & 9.898 \\
$F^{4}(f, f)$ & 6.362 & 6.613 & 6.855 & 6.457 \\
$F^{6}(f, f)$ & 4.675 & 4.863 & 5.043 & 4.736 \\
$F^{2}(d, f)$ & 10.648 & 11.082 & 11.496 & 11.007 \\
$F^{4}(d, f)$ & 6.847 & 7.151 & 7.442 & 7.078 \\
$G^{1}(d, f)$ & 12.549 & 13.139 & 13.701 & 13.023 \\
$G^{3}(d, f)$ & 7.764 & 8.136 & 8.491 & 8.048 \\
$G^{5}(d, f)$ & 5.541 & 5.811 & 6.067 & 5.742 \\
\hline \hline
\end{tabular}

Centre, Karlsruhe. Since these samples emit $\alpha$ particles, they are confined between double-wall containers formed by an internal aluminium holder with a beryllium window protected by a 10-micron-thick kapton foil. For $\mathrm{UO}_{2}$ a single crystal was used with an external surface perpendicular to a [111] direction, and was without a Be window.

The NIXS measurements were performed using the ID16 inverse-geometry, multiple-analyzer-crystal spectrometer at the European Synchrotron Radiation Facility in Grenoble, France $[50,51]$. The beam generated by three consecutive undulators was monochromatized by a $\mathrm{Si}(111)$ double crystal, and horizontally focused by a Rh-coated mirror. The beam size at the sample position was $0.3 \times 1.3 \mathrm{~mm}^{2}$ (horizontal $\times$ vertical). A set of nine spherically bent $\operatorname{Si}(660)$ analyzer crystals with $1 \mathrm{~m}$ bending radius, horizontal scattering geometry, and vertical Rowland circles provided a bandwidth of $1.3 \mathrm{eV}$ at a final photon energy $E_{f}=9.689 \mathrm{keV}$, and an intensity of $7 \times 10^{13}$ photons s$^{-1}$ for a $25 \mu \mathrm{rad}$ vertical divergence of the undulator radiation. The Bragg angle of the analyzers was fixed at $88.5^{\circ}$, and the geometry chosen in order to measure two different momentum-transfer values simultaneously ( $q$ at 9 and $12 \AA^{-1}$ ) using a position-sensitive detector based on a $256 \times 256$ photon-counting pixel array.

Data were collected in symmetric reflection geometry by scanning the incident photon energy $\hbar \omega_{i}=\hbar \omega_{f}+\hbar \omega$ at fixed final energy, covering the $\hbar \omega$ energy-loss interval corresponding to the actinide $O_{4,5}$ absorption edges. The obtained NIXS spectrum for $\mathrm{UO}_{2}$ was consistent with previously published isotropic spectra $[19,20]$. A photon energy-loss spectrum at room temperature over an extended energy range including the elastic and Compton peaks can be found elsewhere [20].

With the aid of the imaging capability of the spherically bent crystal analyzers $[52,53]$ we could efficiently separate the photons scattered by the sample environment, i.e., the

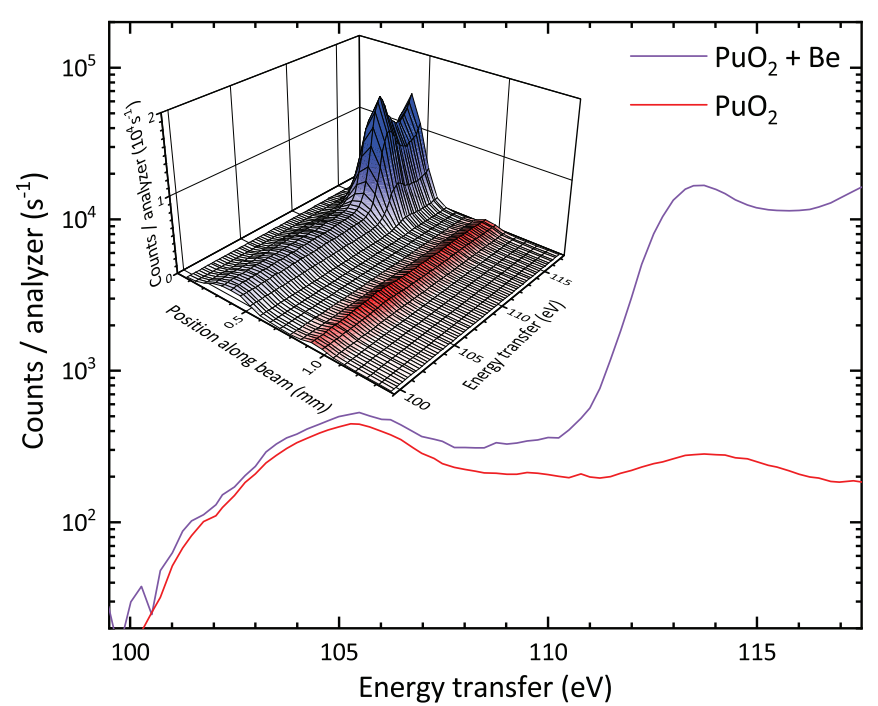

FIG. 2. Semilog plot of the $\mathrm{PuO}_{2}$ spectrum at $q=9.1 \AA^{-1}$ using the $\mathrm{Si}(660)$ crystal analyzer, before (purple) and after (red) background subtraction. The inset shows a linear plot of the signals registered on the position-sensitive detector. Sample and Be cover are separated by $\sim 0.5 \mathrm{~mm}$ in their spatial positions. The Be signal is roughly two orders of magnitude stronger than that from the sample. Despite this image separation, the extremely strong signal from the Be cover and its associated extended x-ray absorption fine structure beyond the $K$ edge suggest that the $\mathrm{PuO}_{2}$ signal cannot be fully trusted to be completely clean for energy transfer beyond $112 \mathrm{eV}$.

beryllium encapsulation and air, and obtain the spectrum of photons scattered by the $\mathrm{Np}$ and $\mathrm{Pu}$ samples only. This was found crucial in order to avoid the encapsulating Be $K$ edge contribution (at $113.4 \mathrm{eV}$ energy transfer) to the measured spectra-otherwise the measured spectra would have been completely swamped by the background signal from the encapsulation. Figure 2 shows the $\mathrm{PuO}_{2}$ spectrum, which was measured at $q=9.1 \AA^{-1}$ using the $\operatorname{Si}(660)$ crystal, before and after background correction. The inset shows that the $\mathrm{Be}$ contribution, with its first peak at $113.4 \mathrm{eV}$, is at a different spatial position along the beam, and hence can be excluded by using imaging capabilities of the technique arising from the position of the signals on the multidetector.

Nevertheless, after Be correction there remained a small bump at $\sim 114 \mathrm{eV}$ (see Fig. 3), which may be caused by a low amount of remnant of the tail of the Be signal. The region above $112 \mathrm{eV}$ was therefore not considered in the fitting of the $\mathrm{Pu}$ compound data. Note that the $\mathrm{UO}_{2}$ data in Fig. 3 are clean since this material requires no Be window. We emphasize that the background correction is a matter of the geometry of the encapsulation. A wider capsule with a larger distance between sample and window will make it easier to remove the $\mathrm{Be}$ signal, so this method can be perfected.

\section{RESULTS AND DISCUSSION}

\section{A. Experimental results}

The $O_{4,5}$ NIXS spectra measured at $q=9$ and $12 \AA^{-1}$ are shown in Fig. 3 by black and gray dots, respectively. $\mathrm{UO}_{2}$ was only measured at $q=9 \AA^{-1}$ as it has been extensively 


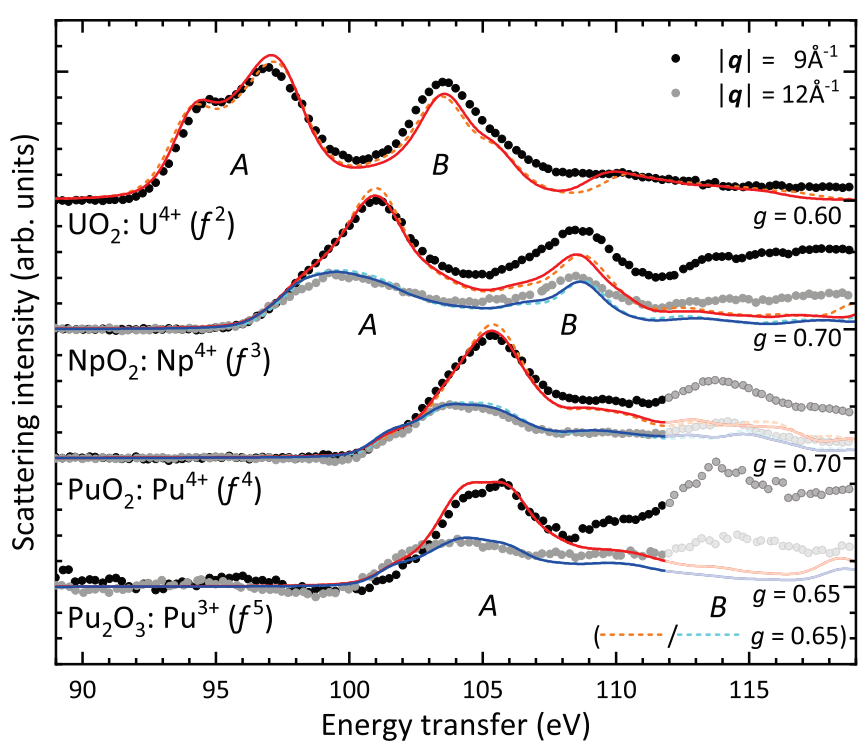

FIG. 3. NIXS $5 d \rightarrow 5 f$ spectra for $\mathrm{UO}_{2}, \mathrm{NpO}_{2}, \mathrm{PuO}_{2}$, and $\mathrm{Pu}_{2} \mathrm{O}_{3}$ measured at $q=9 \AA^{-1}$ (black dots) and $12 \AA^{-1}$ (gray dots), together with simulated spectra calculated at $q=9.5 \AA^{-1}$ (red lines) and $13 \AA^{-1}$ (blue lines) for indicated $g$ values. Corresponding dashed lines are for $g=0.65$, kept fixed for all compounds. The dimmed region above $112 \mathrm{eV}$ for $\mathrm{Pu}$ is where the background correction for the Be foil was performed.

reported before [20] and figures here for comparison. Most noticeable in the spectra is the shift in average energy for the different actinides. As in XAS, the core-level binding energy increases with the atomic number of the element. The average energies $E_{\text {av }}$ of the configurations used to calculate the spectra are given in Table II. Due to the attractive interaction between $5 d$ core hole and $5 f$ electrons, the $\mathrm{Pu} 5 f^{5} \rightarrow$ $5 d^{9} 5 f^{6}$ transition is found at slightly lower energy than the $\mathrm{Pu} 5 f^{4} \rightarrow 5 d^{9} 5 f^{5}$, as was also evidenced by $4 f$ core-level photoemission [54].

The spectra are split into two structures denoted as peaks $A$ and $B$. Although tempting, it would not be correct to denote these separate peak structures as $O_{5}$ and $O_{4}$, respectively. In contrast to deep core levels, these two structures do not simply correspond to transitions from core levels with angular momenta $j=5 / 2$ and $j=3 / 2$, respectively, but instead are strongly mixed in $j$. The observed energy splitting in the $O_{4,5}$ spectrum is primarily due to multiplet splitting by the $5 d-5 f$ electrostatic interaction $[8,11,12]$. As seen in Table II, the exchange interaction $G^{1}(5 d, 5 f) \approx 13 \mathrm{eV}$ exceeds the spin-orbit interaction $\zeta(5 d) \approx 3 \mathrm{eV}$ that gives a splitting of $\frac{5}{2} \zeta(5 d)$.

The spectra for $q=12 \AA^{-1}$ are found at a lower averaged energy compared to $9 \AA^{-1}$ due to the increased $k=5$ contribution, which allows transitions to lower energy states (cf. Fig. 4). While the measured structure for $q=12 \AA^{-1}$ is less pronounced, for $q=9 \AA^{-1}$ the peak $A$ displays a remarkably varied peak shape across the compounds. In $\mathrm{UO}_{2}$ the leading shoulder at $\sim 95 \mathrm{eV}$ arises mainly from $k=5$ transitions, whereas the peaks at 97 and $104 \mathrm{eV}$ arise from both $k=3$ and $k=5$ transitions. For peak $A$ the distinct double structure of $\mathrm{UO}_{2}$ morphs into a single structure for

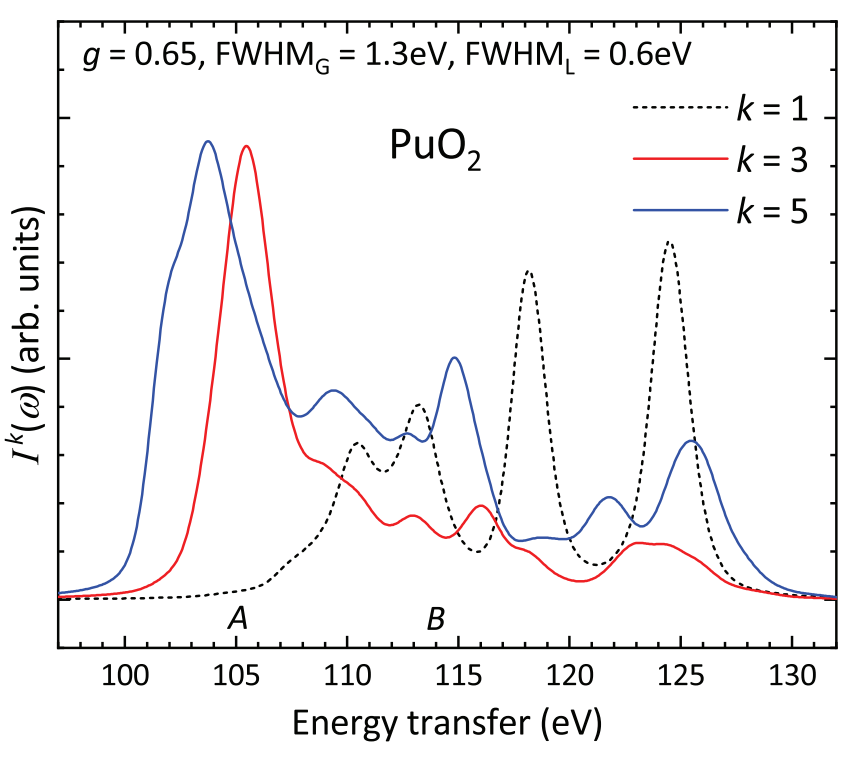

FIG. 4. The calculated $I_{k}$ spectra, i.e., the separate contributions to the cross section given in Eq. (5), for the dipole $(k=1)$, octupole $(k=3)$, and triakontadipole $(k=5)$ transitions of the $\mathrm{Pu}^{4+} 5 d \rightarrow 5 f$ NIXS. The labels $A$ and $B$ mark the energy positions of the two peak structures of the $\mathrm{PuO}_{2}$ spectrum in Fig. 3.

$\mathrm{NpO}_{2}$ and $\mathrm{PuO}_{2}$ and changes back into a twinned structure for $\mathrm{Pu}_{2} \mathrm{O}_{3}$. Comparing the $\mathrm{PuO}_{2}$ and $\mathrm{Pu}_{2} \mathrm{O}_{3}$ data, the rather different multiplet structures of peak $A$ qualify to determine the bulk $5 f$-electron count.

\section{B. Calculated results}

The calculated fits are shown in Fig. 3 by the drawn lines, which give good overall agreement for peak $A$. However, the peak $B$ intensity in the $\mathrm{Pu} 5 f^{4}$ and $5 f^{5}$ calculations is much lower than in the measured spectra, which reveal a bump at $\sim 114 \mathrm{eV}$. However, previous calculations [11] have decisively shown that $5 f^{n}$ configurations with $n>3$ have a low-intensity peak $B$. Staying within a realistic physical parameter choice, it turns out to be impossible to increase the peak $B$ intensity to the level as seen in the experimental spectra. One could drastically increase the ratio of $\zeta(5 d) / G^{1}(5 d, 5 f)$ but that brings with it the undesired consequence of losing the structure within peak $A$ [11]. Moreover, it would violate the long established picture of intermediate coupling for atomic spectra [48]. A crystal-field interaction can mix the different $J$ levels and according to the NIXS sum rule [23] this would increase the relative intensity of peak $B$. However, the $5 f$ crystal-field interaction is too weak to explain the large discrepancy between the calculated and measured intensities of peak $B$. Whereas the $\mathrm{Be} K$ peak at $113.5 \mathrm{eV}$ has been mostly removed, a remnant amount of Be signal of $\sim 1 \%$ may possibly give such a contribution. Therefore, for the two $\mathrm{Pu}$ spectra, only peak $A$ in Fig. 3 was included in the fit.

After initially performing the fits using the experimental values of $q=9$ and $12 \AA^{-1}$, the $k=5$ spectral contribution, which is prominent at the low-energy side of peak $A$, was found to be too small for all compounds; i.e., the ratio $(k=$ $5) /(k=3)$ was too low. As this ratio is fixed by the value 
TABLE III. Calculated NIXS scattering cross sections $\left(\times 10^{3}\right)$ of the $5 d \rightarrow 5 f$ transitions for the multipole moments $k$ at specific $q$ values (in $\AA^{-1}$ ); cf. Fig. 1 .

\begin{tabular}{lcccc}
\hline \hline & $q=9$ & $q=9.5$ & $q=12$ & $q=13$ \\
\hline$k=1$ & 1.53 & 0.42 & 0.33 & 0.25 \\
$k=3$ & 18.87 & 16.60 & 5.02 & 2.31 \\
$k=5$ & 5.52 & 6.31 & 7.79 & 7.02 \\
$(k=5) /(k=3)$ & 0.293 & 0.380 & 1.552 & 3.039 \\
\hline \hline
\end{tabular}

of $q$, we increased $q$ in the calculation to 9.5 and $13 \AA^{-1}$, respectively, resulting in a much better agreement with experiment for all compounds. Actually, an increased $q$ value was already used in a previous calculation for $\mathrm{UO}_{2}$ [28]. A change in $q$ changes the relative intensities of the $k=5,3$, and 1 spectra, while each of these $k$ spectra keeps the same shape. Figure 4 shows the calculated individual spectra of $\mathrm{PuO}_{2}$ for each $k$; for the other actinides they can be found elsewhere [11]. To obtain the intensity of these spectra they have to be multiplied by the scattering cross sections, which are shown in Fig. 1 for the different multipole moments $k$. They are also listed in Table III for the specific $q$ values. The table shows that changing $q$ from 9 to $9.5 \AA^{-1}$ increases the scattering ratio for the $(k=5) /(k=3)$ spectra by $30 \%$, and changing $q$ from 12 to $13 \AA^{-1}$ increases it by $96 \%$. For larger $q$ the $k=1$ contribution can be omitted.

Interestingly, this increase in the effective $q$ corresponds to a contraction of the radial $5 f$ wave function. Whereas the core hole is accounted for at an atomic level, the strongest effect that is missing is the interatomic hybridization in the solid state that alters the wave function such that one has to adjust both the electrostatic interactions and $q$. This adjustment appears to be larger for the Slater integrals $(g=$ $0.6-0.7)$ than for $q(5 \%-12 \%$ increase). Spin-orbit interaction is not significantly affected because as a relativistic effect it mostly originates from close to the core, whereas the change is mainly occurring in the overlapping part of the wave function.

Using a fixed value of the reduction factor $g$ of the Slater integrals for all compounds resulted in an optimal value of $g=0.65$. Such a value is in agreement with previous simulations for $\mathrm{UO}_{2}$ measured with a higher energy resolution [26]. Removing the constraint of having a single value of $g$ for all compounds resulted in values between 0.60 and 0.70 , as listed in Table I. Both of these fits are shown in Fig. 3 but are very similar.

\section{CONCLUSIONS}

The experimental NIXS spectra at the actinide $O_{4,5}$ edge show a good agreement with multiplet calculations by reducing the Slater integrals of $5 d-5 f$ Coulomb and exchange integrals by $g=0.6-0.7$ with respect to their Hartree-Fock values. Comparing the $\mathrm{PuO}_{2}$ and $\mathrm{Pu}_{2} \mathrm{O}_{3}$ spectra, the different multiplet structures of the $O_{5}$ edges qualify to determine the bulk $5 f$-electron count.
The spectral shape depends strongly on the momentum transfer $q$, which controls the relative contributions of the multipole transitions $k=3$ and 5. In order to obtain good agreement, the momentum transfer $q$ in the calculation had to be increased compared to the experiment, which corresponds to a contraction of the atomic wave functions. Such a change is expected when going from the calculated atomic wave functions to the hybridized wave functions in the solid state. Recent theoretical studies suggest a strong $5 f-\mathrm{O} 2 p$ orbital mixing in actinide oxides [55,56]. Spectra for such an orbital mixing can in principle be calculated by taking a coherent state $5 f^{n}+5 f^{n+1} \underline{L}$, where $\underline{L}$ denotes a ligand hole state, as done, e.g., for Pu $4 f$ photoemission spectroscopy (PES) [54]. However, in the case of PES there are strong satellites due to the presence of the unscreened core hole in the final state, which rearranges the $5 f$ energy levels after photoemission. In XAS and NIXS the core hole potential is largely screened by the extra $5 f$ electron and any satellite structure would be fairly weak. Using the spectral profile of the current data to evidence covalent mixing would be a challenging task. However, future data of improved quality could expose the presence of covalent mixing.

Despite the difficulty of working with transuranium samples at large user facilities, NIXS experiments have considerable promise to further our understanding of these important materials. First, better experimental facilities now exist since these experiments were performed [50]. Second, Be encapsulation can be avoided and kapton used, as long as the experiments are conducted only at room temperature. Third, the sensitivity to the spatial extent of the wave functions suggests that the oxides through to $\mathrm{AmO}_{2}$ should be examined, and at the same time, an effort to measure samples of $\alpha$-Pu and Am metal should be made. This would give a much higher level of understanding of the wave function issue, as it is known that between $\alpha$-Pu and Am there is an almost $40 \%$ change in the atomic volume [8], which should be immediately evident in the position, structure, and intensity of the spectra.

Our results demonstrate the feasibility to measure higher multipole moment transitions of transuranium compounds. The higher multipole moments allow us to fit a set of different spectra, instead of a single spectrum for the dipole transition. Multipole transitions are expected to become readily available from future high-energy x-ray sources, which can lead to new ways of doing spectroscopy $[57,58]$. As the photonmatter interaction is reasonably strong for shallow core levels, NIXS requires only small sample volumes, compared to, e.g., neutron scattering. This enables us to do NIXS on very small volume samples, thin films, buried interfaces, and nanoobjects.

\section{ACKNOWLEDGMENTS}

Experiments were done on beamline ID16 at the European Synchrotron Radiation Facility (ESRF) in Grenoble, France, under Proposal No. HC-3455. We would like to thank Maurits Haverkort for making the QUANTY code available and Andrea Severing for discussions. M.S. benefited from the financial support of the Deutsche Forschungsgemeinschaft (DFG) under Grant No. SE-1441/5-1. S.H. was supported by the Academy of Finland (Grant No. 1295696). 
[1] G. van der Laan and A. I. Figueroa, Coord. Chem. Rev. 277278, 95 (2014).

[2] G. van der Laan and I. W. Kirkman, J. Phys.: Condens. Matter 4, 4189 (1992).

[3] B. T. Thole, G. van der Laan, J. C. Fuggle, G. A. Sawatzky, R. C. Karnatak, and J. M. Esteva, Phys. Rev. B 32, 5107 (1985).

[4] H. Ogasawara, A. Kotani, and B. T. Thole, Phys. Rev. B 44, 2169 (1991).

[5] G. van der Laan, J. Phys.: Condens. Matter 3, 7443 (1991).

[6] K. Starke, E. Navas, E. Arenholz, Z. Hu, L. Baumgarten, G. van der Laan, C. T. Chen, and G. Kaindl, Phys. Rev. B 55, 2672 (1997).

[7] J. Terry, R. K. Schulze, J. D. Farr, T. Zocco, K. Heinzelmann, E. Rotenberg, D. K. Shuh, G. van der Laan, D. A. Arena, and J. G. Tobin, Surf. Sci. 499, L141 (2002).

[8] K. T. Moore and G. van der Laan, Rev. Mod. Phys. 81, 235 (2009).

[9] J. L. Dehmer, A. F. Starace, U. Fano, J. Sugar, and J. W. Cooper, Phys. Rev. Lett. 26, 1521 (1971).

[10] B. T. Thole and G. van der Laan, Phys. Rev. B 38, 3158 (1988).

[11] G. van der Laan, Phys. Rev. B 86, 035138 (2012).

[12] K. T. Moore and G. van der Laan, Ultramicroscopy 107, 1201 (2007).

[13] J. A. Soininen, A. L. Ankudinov, and J. J. Rehr, Phys. Rev. B 72, 045136 (2005).

[14] B. C. Larson, W. Ku, J. Z. Tischler, C.-C. Lee, O. D. Restrepo, A. G. Eguiluz, P. Zschack, and K. D. Finkelstein, Phys. Rev. Lett. 99, 026401 (2007).

[15] M. W. Haverkort, A. Tanaka, L. H. Tjeng, and G. A. Sawatzky, Phys. Rev. Lett. 99, 257401 (2007).

[16] R. A. Gordon, G. T. Seidler, T. T. Fister, M. W. Haverkort, G. A. Sawatzky, A. Tanaka, and T. K. Sham, Europhys. Lett. 81, 26004 (2008).

[17] T. T. Fister, F. D. Vila, G. T. Seidler, L. Svec, J. C. Linehan, and J. O. Cross, J. Am. Chem. Soc. 130, 925 (2008).

[18] R. Gordon, M. Haverkort, S. Sen Gupta, and G. Sawatzky, J. Phys.: Conf. Ser. 190, 012047 (2009).

[19] J. A. Bradley, S. Sen Gupta, G. T. Seidler, K. T. Moore, M. W. Haverkort, G. A. Sawatzky, S. D. Conradson, D. L. Clark, S. A. Kozimor, and K. S. Boland, Phys. Rev. B 81, 193104 (2010).

[20] R. Caciuffo, G. van der Laan, L. Simonelli, T. Vitova, C. Mazzoli, M. A. Denecke, and G. H. Lander, Phys. Rev. B 81, 195104 (2010).

[21] J. A. Bradley, K. T. Moore, G. van der Laan, J. P. Bradley, and R. A. Gordon, Phys. Rev. B 84, 205105 (2011).

[22] J. A. Bradley, A. Sakko, G. T. Seidler, A. Rubio, M. Hakala, K. Hämäläinen, G. Cooper, A. P. Hitchcock, K. Schlimmer, and K. P. Nagle, Phys. Rev. A 84, 022510 (2011).

[23] G. van der Laan, Phys. Rev. Lett. 108, 077401 (2012).

[24] T. Willers, F. Strigari, N. Hiraoka, Y. Q. Cai, M. W. Haverkort, K.-D. Tsuei, Y. F. Liao, S. Seiro, C. Geibel, F. Steglich, L. H. Tjeng, and A. Severing, Phys. Rev. Lett. 109, 046401 (2012).

[25] M. Sundermann, M. W. Haverkort, S. Agrestini, A. Al-Zein, M. Moretti Sala, Y. Huang, M. Golden, A. de Visser, P. Thalmeier, L. H. Tjeng, and A. Severing, Proc. Natl. Acad. Sci. U.S.A. 113, 13989 (2016).

[26] M. Sundermann, H. Yavas, K. Chen, D. J. Kim, Z. Fisk, D. Kasinathan, M. W. Haverkort, P. Thalmeier, A. Severing, and L. H. Tjeng, Phys. Rev. Lett. 120, 016402 (2018).
[27] S. Sen Gupta, J. A. Bradley, M. W. Haverkort, G. T. Seidler, A. Tanaka, and G. A. Sawatzky, Phys. Rev. B 84, 075134 (2011).

[28] M. Sundermann, G. van der Laan, A. Severing, L. Simonelli, G. H. Lander, M. W. Haverkort, and R. Caciuffo, Phys. Rev. B 98, 205108 (2018).

[29] B. Ao, H. Lu, Z. Yang, R. Qiu, and S.-X. Hu, Phys. Chem. Chem. Phys. 21, 4732 (2019).

[30] G. Amoretti, A. Blaise, R. Caciuffo, J. M. Fournier, M. T. Hutchings, R. Osborn, and A. D. Taylor, Phys. Rev. B 40, 1856 (1989).

[31] J. M. Fournier, A. Blaise, G. Amoretti, R. Caciuffo, J. Larroque, M. T. Hutchings, R. Osborn, and A. D. Taylor, Phys. Rev. B 43, 1142 (1991).

[32] S. Kern, R. A. Robinson, H. Nakotte, G. H. Lander, B. Cort, P. Watson, and F. A. Vigil, Phys. Rev. B 59, 104 (1999).

[33] N. Magnani, P. Santini, G. Amoretti, and R. Caciuffo, Phys. Rev. B 71, 054405 (2005).

[34] P. Santini, S. Carretta, G. Amoretti, R. Caciuffo, N. Magnani, and G. H. Lander, Rev. Mod. Phys. 81, 807 (2009).

[35] J. A. Paixão, C. Detlefs, M. J. Longfield, R. Caciuffo, P. Santini, N. Bernhoeft, J. Rebizant, and G. H. Lander, Phys. Rev. Lett. 89, 187202 (2002).

[36] N. Magnani, S. Carretta, R. Caciuffo, P. Santini, G. Amoretti, A. Hiess, J. Rebizant, and G. H. Lander, Phys. Rev. B 78, 104425 (2008).

[37] S. B. Wilkins, R. Caciuffo, C. Detlefs, J. Rebizant, E. Colineau, F. Wastin, and G. H. Lander, Phys. Rev. B 73, 060406(R) (2006).

[38] S. Carretta, P. Santini, R. Caciuffo, and G. Amoretti, Phys. Rev. Lett. 105, 167201 (2010).

[39] R. Caciuffo, P. Santini, S. Carretta, G. Amoretti, A. Hiess, N. Magnani, L.-P. Regnault, and G. H. Lander, Phys. Rev. B 84, 104409 (2011).

[40] G. Raphael and R. Lallement, Solid State Commun. 6, 383 (1968).

[41] H. Yasuoka, G. Koutroulakis, H. Chudo, S. Richmond, D. K. Veirs, A. I. Smith, E. D. Bauer, J. D. Thompson, G. D. Jarvinen, and D. L. Clark, Science 336, 901 (2012).

[42] A. B. Shick, J. Kolorenč, L. Havela, T. Gouder, and R. Caciuffo, Phys. Rev. B 89, 041109(R) (2014).

[43] M. Wulff and G. H. Lander, J. Chem. Phys. 89, 3295 (1988).

[44] I. D. Prodan, G. E. Scuseria, J. A. Sordo, K. N. Kudin, and R. L. Martin, J. Chem. Phys. 123, 014703 (2005).

[45] I. D. Prodan, G. E. Scuseria, and R. L. Martin, Phys. Rev. B 73, 045104 (2006).

[46] G. Jomard, B. Amadon, F. Bottin, and M. Torrent, Phys. Rev. B 78, 075125 (2008).

[47] L. Petit, A. Svane, Z. Szotek, W. M. Temmerman, and G. M. Stocks, Phys. Rev. B 81, 045108 (2010).

[48] R. D. Cowan, The Theory of Atomic Structure and Spectra (University of California Press, Berkeley, 1981).

[49] M. W. Haverkort, J. Phys.: Conf. Ser. 712, 012001 (2016).

[50] The inelastic scattering beamline ID16 was replaced by the new IXS beamline ID20 [S. Huotari, Ch. J. Sahle, Ch. Henriquet, A. Al-Zein, K. Martel, L. Simonelli, R. Verbeni, H. Gonzalez, M.-C. Lagier, C. Ponchut, M. Moretti Sala, M. Krisch, and G. Monaco, J. Synchrotron Radiat. 24, 521 (2017)].

[51] R. Verbeni, T. Pylkkänen, S. Huotari, L. Simonelli, G. Vankó, K. Martel, C. Henriquet, and G. Monaco, J. Synchrotron Radiat. 16, 469 (2009). 
[52] S. Huotari, T. Pylkkänen, R. Verbeni, G. Monaco, and K. Hämäläinen, Nat. Mater. 10, 489 (2011).

[53] C. J. Sahle, A. Mirone, T. Vincent, A. Kallonen, and S. Huotari, J. Synchrotron Radiat. 24, 476 (2017).

[54] G. van der Laan and M. Taguchi, Phys. Rev. B 82, 045114 (2010).

[55] I. D. Prodan, G. E. Scuseria, and R. L. Martin, Phys. Rev. B 76, 033101 (2007).

[56] X.-D. Wen, R. L. Martin, L. E. Roy, G. E. Scuseria, S. P. Rudin, E. R. Batista, T. M. McCleskey, B. L. Scott, E. Bauer,
J. J. Joyce, and T. Durakiewicz, J. Chem. Phys. 137, 154707 (2012).

[57] K.-D. Liss, A. Bartels, A. Schreyer, and H. Clemens, Textures Microstruct. 35, 219 (2003).

[58] N. Schell, A. King, F. Beckmann, H.-U. Ruhnau, R. Kirchhof, R. Kiehn, M. Müller, and A. Schreyer, in 10th International Conference on Radiation Instrumentation SRI 2009, edited by R. Garrett, I. Gentle, K. Nugent, and S. Wilkins, AIP Conf. Proc. No. 1234 (AIP, New York, 2010), p. 391. 